\title{
Controle de Flebotomíneos com DDT, em Área Endêmica de Leishmaniose Tegumentar no Estado do Paraná, Sul do Brasil.
}

\author{
Ueslei Teodoro ${ }^{*}$, Eunice A.B. Galati ${ }^{2}$, João Balduíno Kühl ${ }^{1}$, Ana Leuch Lozovei ${ }^{3}$ e Orlando Carlos \\ Barbosa $^{4}$ \\ ${ }^{1}$ Laboratório de Parasitologia Básica do Departamento de Análises Clínicas, Centro de Ciências da Saúde, \\ Universidade Estadual de Maringá. Av. Colombo, 5790. Maringá - PR, Brasil - CEP 87020-900. ${ }^{2}$ Departamento de \\ Epidemiologia, Faculdade de Saúde Pública, Universidade de São Paulo. Av. Dr Arnaldo, $715 .{ }^{3}$ Departamento de \\ Patologia Básica da Universidade Federal do Paraná, Centro Politécnico, Curitiba, PR, Brasil. ${ }^{4}$ Fundação \\ Nacional de Saúde do Ministério da Saúde - Londrina, PR, Brasil.
}

\begin{abstract}
Evaluation of DDT insecticide used to sand flies control in domiciles and domestic animal shelters in an endemic area of cutaneous leishmaniasis. Collections of sand flies were made with Falcão traps in houses, in one pigsty and in one tree in Jussara Farm, municipality of Jussara, State of Paraná, Brazil, between 10 p.m. and 2 a.m. during 7 days from March 24 to April 1, 1992. In May, 1992 DDT insecticide (Dicloro Difenil Tricloro Etano) (1 g/square meter of area) was applied internally and externally on houses and in pigsty. After this, new collections of sand flies were made from July 1992 to February 1993, once a month, with Falcão traps installed in the same hour and conditions mentioned above. Before DDT insecticide application 12,960 sand flies were collected (Hourly Average $=463$ ) and it was observed the prevalence (61,8\%). of Lutzomyia (Nyssomyia) whitmani (Antunes \& Coutinho, 1939) After the DDT application only 589 sand flies were collected (Hourly Average =18,4) and also L. whitmani predominated again $(90,8 \%)$. The decrease of sand flies number after the DDT insecticide application in domiciles and in pigsty prove that this measure can help cutaneous leishmaniasis control in Northern Paraná.
\end{abstract}

Palavras-chave: Leishmania; leishmaniose; controle de flebotomíneos; DDT

Key words: Leishmania; leishmaniasis; sand flies control. DDT insecticide

\section{INTRODUÇÃO}

A leishmaniose tegumentar tem-se manifestado em quase todo o território brasileiro de forma crescente, ano a ano, seja pela melhoria da notificação seja pelo aumento do número de casos, ou por ambos os motivos. Nos últimos vinte anos o número de casos notificados superou 300 mil (MINISTÉRIO DA SAÚDE, 1988;1995; 1996).

No Estado do Paraná, a leishmaniose tegumentar vem sendo notificada, especialmente, a partir de 1980 (MINISTÉRIO DA SAÚDE, 1988; 1995; 1996). No município de Jussara, localizado na região noroeste do Estado do Paraná, a leishmaniose tegumentar é endêmica, atingindo a população humana e canina
Silveira et al, 1996a; Silveira et al, 1996b). Neste município, Lonardoni et al (1993) isolaram Leishmania (Viannia) braziliensis Vianna, 1911 de cães, na Fazenda Jussara.

O uso do DDT para o controle de flebotomíneos na América foi feito primeiramente no Peru (Hertig \& Fairchild, 1948). No Brasil, o emprego do DDT como medida de controle de flebotomíneos comprovou a eficácia deste inseticida reduzindo progressivamente a transmissão de leishmanioses (Alencar, 1961; Deane; Deane, \& Alencar, 1955; Magalhães et $l$, 1980; Nery-Guimarães \& Bustamante, 1954; Sherlock \& Almeida, 1970).

\footnotetext{
* Autor para correspondência
} 
No Estado do Paraná o emprego de medidas profiláticas que ajudem a interromper o ciclo de transmissão de Leishmania é necessário e urgente, especialmente no domicílio e peridomicílio de áreas onde o risco de infecção tem sido elevado. Com este objetivo desenvolveram-se estudos para a avaliação do impacto do DDT no controle da leishmaniose tegumentar na Fazenda Jussara, município de Jussara, cujos resultados são aqui apresentados.

\section{MATERIAL E MÉTODOS}

1. Descrição da área.

O município de Jussara localiza-se entre $23^{\circ} 32^{\prime}$ e $23^{\circ} 36^{\prime}$ latitude Sul e $52^{\circ} 29^{\prime}$ e $52^{\circ} 23^{\prime}$ longitude Oeste. A área de execução da pesquisa foi descrita anteriormente de forma mais detalhada (Teodoro, 1995). A precipitação pluviométrica no decorrer de 1992 foi 1.801 $\mathrm{mm}$, o que dá uma média mensal de $150 \mathrm{~mm}$. O mês de maior precipitação neste ano foi maio com 336,5 mm e o de menor junho com 23,5 mm. Em 1993, a precipitação total foi de 1.538 $\mathrm{mm}$, dando uma média mensal de $128,2 \mathrm{~mm}$. A temperatura média anual, em 1992 , foi $23,7{ }^{\circ} \mathrm{C}$, com a média mais baixa de $18,6{ }^{\circ} \mathrm{C}$, em julho e a mais elevada de $27{ }^{\circ} \mathrm{C}$, em dezembro. Em 1993, a temperatura média anual foi $23,6{ }^{\circ} \mathrm{C}$, a média mais baixa ocorreu em junho $\left(17,9{ }^{\circ} \mathrm{C}\right)$ e a média mais alta em janeiro $\left(27,2^{\circ} \mathrm{C}\right)$.

2. Coletas de flebotomíneos anterior à dedetização.

$\mathrm{Na}$ Fazenda Jussara, os flebotomíneos foram capturados com armadilhas de Falcão (Falcão, 1981) no período compreendido entre 24 de março e $1^{\circ}$ de abril de 1992 , em 7 noites, das 22 às 02 horas, somando 28 horas de coletas por armadilha. Uma armadilha $(\mathrm{G})$ foi instalada dentro de uma residência, situada ao lado da guarita, na entrada da fazenda, onde uma mulher adulta e dois de seus filhos ainda crianças estavam com leishmaniose tegumentar (Figura 1). Aproximadamente, a 1.250 metros desta guarita, ao lado de um viveiro de mudas para reflorestamento, quatro armadilhas foram instaladas conforme a seguir: a primeira (R1) dentro da residência 1 , a segunda (R4) na residência 4 , a terceira $(\mathrm{P})$ numa pocilga e a última (A) numa árvore (mangueira) utilizada como poleiro por diversas galinhas (Figura 1).

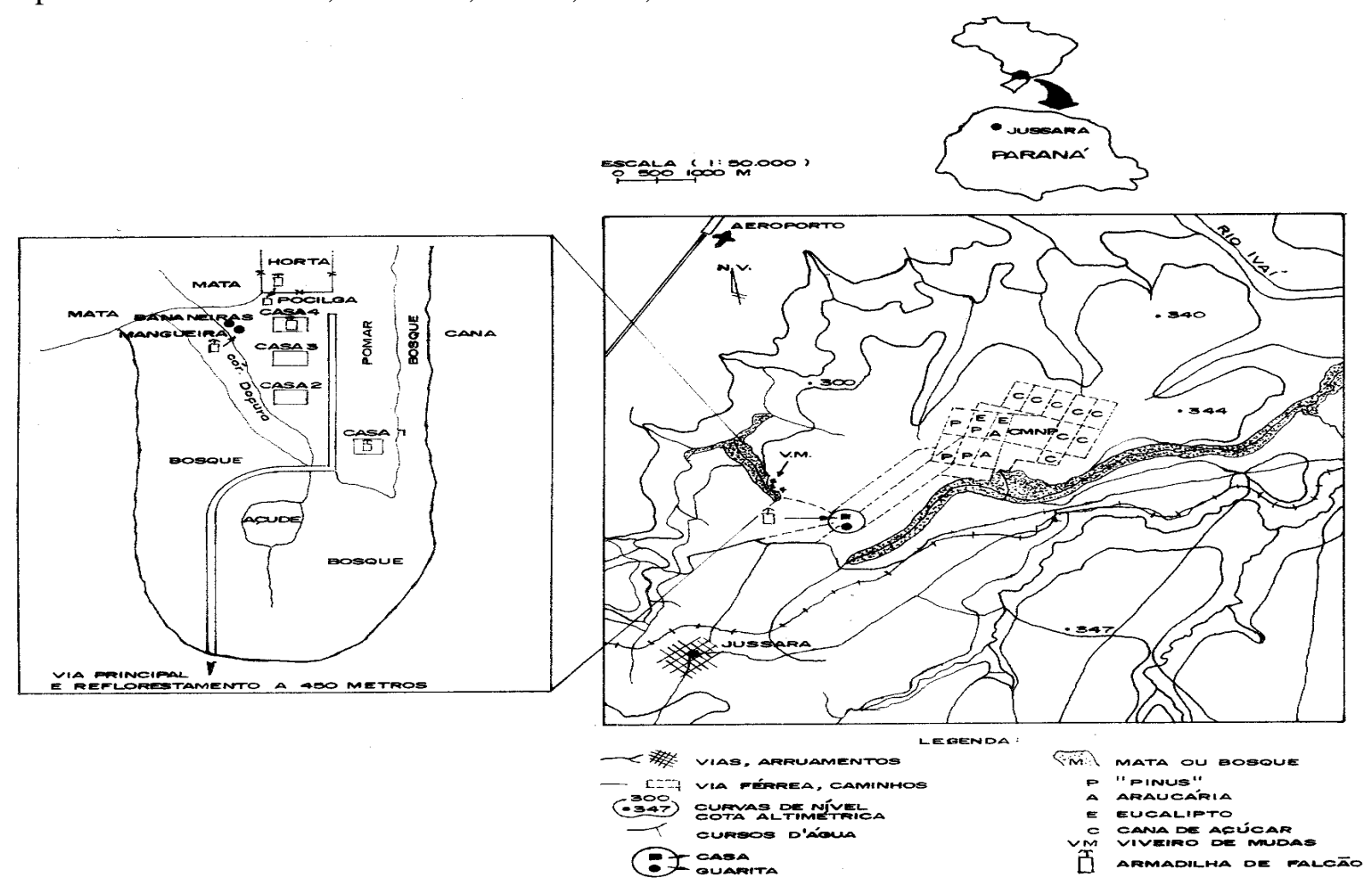

Figura 1. Área onde foi realizada a pesquisa e locais de instalação das armadilhas de Falcão na Fazenda Jussara, município de Jussara, Paraná, Brasil. 
3. Dedetização dos domicílios e da pocilga.

No mês de maio de 1992, sob o comando da Fundação Nacional de Saúde, as superfícies internas e externas das paredes das residências e da pocilga supramencionadas foram dedetizadas (1g de DDT $/ \mathrm{m}^{2}$ ), com bombas costais tipo Hudson. Optou-se pelo uso do DDT pela sua disponibilidade na Fundação Nacional de Saúde e pela sua reconhecida eficácia no controle de flebotomíneos no domicílio e peridomicílio.

Na residência ao lado da guarita foi feita nova dedetização no mês de outubro, a pedido do morador, embora ainda não houvesse indícios de que a quantidade de flebotomíneos estivesse aumentando nesta residência $(\mathrm{G})$. O morador da residência 4 deixou de criar suínos, ficando a pocilga abandonada, logo após a dedetização no mês de maio.

4. Coletas de flebotomíneos posterior à dedetização.

De junho de 1992 a fevereiro de 1993, exceto em agosto, foram realizadas coletas mensais de flebotomíneos, com 5 armadilhas instaladas nos mesmos locais já mencionados, num total de 8 noites, das 22 às 02 horas, somando 32 horas de capturas por armadilha.

\section{RESULTADOS}

No período de 24 de março a $1^{\circ}$ de abril de 1992 foram capturados 12.960 flebotomíneos machos e fêmeas das seguintes espécies: 1) Brumptomyia brumpti (Larrousse, 1920); 2) Lutzomyia firmatoi (Barretto, Martins \& Pellegrino, 1956); 3) Lutzomyia (Pintomyia) fischeri (Pinto, 1926); 4) Lutzomyia (Nyssomyia) intermedia (Lutz \& Neiva, 1912); 5) Lutzomyia migonei (França, 1920); 6) Lutzomyia misionensis (Castro, 1959); 7) Lutzomyia monticola (Costa Lima, 1932); 8) Lutzomyia (Pintomyia) pessoai (Coutinho \& Barretto, 1940); 9) Lutzomyia shannoni (Dyar,1929); e 10) Lutzomyia (Nyssomyia) whitmani (Antunes \& Coutinho, 1939).

A tabela I mostra que Lutzomyia whitmani prevaleceu $(61,8 \%)$ sobre as demais espécies no conjunto das armadilhas e em cada uma delas isoladamente, exceto em R1. Nas armadilhas P e $\mathrm{G}$ foram coletados 7.558 e 4.468 flebotomíneos, respectivamente.

Tabela I. Flebotomíneos coletados dentro de domicílios e numa pocilga na Fazenda Jussara, município de Jussara, Estado do Paraná entre 24 de março e $1^{\circ}$ de abril de 1992, antes da dedetização em maio de 1992.

\begin{tabular}{lccccccc}
\hline \multicolumn{1}{c}{$\begin{array}{c}\text { Armadilha } \\
\text { Espécie }\end{array}$} & $\mathrm{G}$ & $\mathrm{R} 1$ & $\mathrm{R} 4$ & $\mathrm{P}$ & $\mathrm{A}$ & $\mathrm{Total}$ & $\%$ \\
\hline Lutzomyia whitmani & $2 . .351$ & 44 & 145 & 5.033 & 433 & 8.006 & 61,8 \\
Lutzomyia migonei & 1.533 & 4 & 38 & 1.485 & 24 & 3.084 & 23,8 \\
Lutzomyia intermedia & 306 & 45 & 72 & 701 & 80 & 1.204 & 9,3 \\
Lutzomyia fischeri & 156 & 1 & 17 & 243 & 18 & 435 & 3,3 \\
Lutzomyia pessoai & 72 & 1 & 11 & 65 & 1 & 150 & -2 \\
Lutzomyia monticola & 1 & - & - & - & - & 1 & - \\
Lutzomyia firmatoi & 6 & - & - & - & - & 6 & - \\
Lutzomyia shannoni & 8 & - & - & 5 & - & 13 & - \\
Lutzomyia misionensis & - & - & - & 1 & - & 1 & 0,4 \\
Lutzomyia spp. & 35 & - & - & 23 & - & 58 & - \\
Brumptomyia. brumpti & - & - & - & 2 & - & 2 & - \\
\hline \multicolumn{1}{c}{ Total } & 4.468 & 95 & 283 & 7.558 & 556 & 12.960 & \\
\hline
\end{tabular}

$\mathrm{G}=$ Residência ao lado da guarita na entrada da Fazenda Jussara; R1;R4= residências 1 e 4; P= pocilga nos fundos de R1; $\mathrm{A}=$ árvore nos fundos de $\mathrm{R} 4$, onde diversas galinhas empoleiravam-se no período noturno. 
A média horária $(\mathrm{MH})$ de flebotomíneos capturados de 24 de março a $1^{\circ}$ de abril de 1992 foi de 463 (12.960/28).

Na tabela II observa-se que de junho de 1992 a fevereiro de 1993 foram coletados 589 flebotomíneos. Destes 72,5\% (427/589) foram coletados na árvore (armadilha A) aos fundos de R4, não dedetizada. Observou-se maior prevalência de L. whitmani $(90,8 \%)$ após a dedetização em relação aos $61,8 \%$ verificados antes da dedetização (Tabela I).

Tabela II. Flebotomíneos coletados em domicílios e numa pocilga na Fazenda Jussara, município de Jussara, Estado do Paraná, Brasil, de junho de 1992 a fevereiro de 1993, após terem sido dedetizados no mês de maio de 1992.

\begin{tabular}{|c|c|c|c|c|c|c|c|}
\hline Armadilha/Espécie & $\mathrm{G}$ & $\mathrm{R} 1$ & $\mathrm{R} 4$ & $\mathrm{P}$ & $\mathrm{A}$ & Total & $\%$ \\
\hline Lutzomyia whitmani & 5 & 14 & 14 & 94 & 408 & 535 & 90,8 \\
\hline Lutzomyia migonei & 2 & 2 & 2 & 5 & 14 & 25 & 4,2 \\
\hline Lutzomyia intermedia & - & - & - & - & 4 & 4 & 0,7 \\
\hline Lutzomyia fischeri & - & - & - & - & 1 & 1 & 0,2 \\
\hline Lutzomyia spp. & - & - & - & 24 & - & 24 & 4,1 \\
\hline Total & 7 & 16 & 16 & 123 & 427 & 589 & 100,0 \\
\hline$\%$ & 1,2 & 2,7 & 2,7 & 20,9 & 72,5 & 100,0 & \\
\hline
\end{tabular}

$\mathrm{G}=$ Residência ao lado da guarita na entrada da Fazenda Jussara; R1;R4= residências 1 e 4; $\mathrm{P}=$ pocilga nos fundos de R1; A= árvore nos fundos de R4, onde diversas galinhas empoleiravam-se no período noturno.

A média horária de flebotomíneos capturados de junho de 1992 a fevereiro de 1993 foi de 18,4 $(589 / 32)$.

A média horária de 18,4 flebotomíneos coletados após a desinsetização foi $4,0 \%$ da média horária (463). 0 coletada antes.

\section{DISCUSSÃO}

Embora o número de flebotomíneos tenha diminuído significativamente após a dedetização, esta devia ter sido realizada do início de outubro até meados de novembro, pois o efeito residual do DDT seria mais eficaz justamente no período em que a população de flebotomíneos tende a ser maior, na área onde foi realizado este trabalho. A população destes insetos, com predomínio de L. whitmani, no noroeste do Paraná tem sido bem mais elevada nos meses de dezembro, janeiro, fevereiro, março e abril (Teodoro, 1995; Teodoro et al, 1993).

A infecção natural de $L$. whitmani (Azevedo et al, 1990a; Brandão-Filho et al, 1994; Queiroz et al, 1994; Rosa et al, 1988; Vasconcelos et al,
1994) L. migonei (Queiroz et al, 1994; Azevedo et al, 1990b) em ambientes antropogênicos vêm comprovando o potencial vetorial destes flebotomíneos em ambientes naturais.

Os trabalhos de Alencar (1961), Deane et al. (1955), Hertig \& Fairchild (1948), Magalhães et al (1980) , Nery-Guimarães \& Bustamente (1954) e Sherlock \& Almeida (1970) demonstraram que a desinsetização domiciliar e peridomiciliar dão bons resultados no controle da população de vetores de leishmanioses. Contudo, a desinsetização seria mais eficaz se fosse realizada de forma contínua, em época mais apropriada e periodicamente (Alencar, 1983).

A freqüência de flebotomíneos na árvore (mangueira) usada como poleiro de galinhas permaneceu elevada após a dedetização dos domicílios e da pocilga, sugerindo a eficácia da medida profilática, tendo em vista que o DDT não foi aplicado na árvore.

Os resultados mostram que a dedetização contribuiu para reduzir drasticamente o número de flebotomíneos no interior dos domicílios e na 
pocilga. Assim, a desinsetização de domicílios e de abrigos de animais domésticos, com DDT ou outros inseticidas sintéticos, pode ser valiosa para diminuir o número de infecções por Leishmania em áreas endêmicas de leishmaniose tegumentar. Devido a eficácia da desinsetização sugere-se que os serviços de saúde pública passem a promover o controle de flebotomíneos nas áreas endêmicas de leishmanioses, uma vez que o número de casos destas doenças vêm crescendo no Brasil.

\section{AGRADECIMENTOS}

À Fundação Nacional de Saúde (Ministèrio da Saúde) pela execução dos trabalhos de campo. À Cia. Melhoramentos Norte do Paraná, município de Jussara, pelo auxílio inestimável para a execução deste trabalho e ao Sr. Carmelindo Colato, desenhista-projetista do Departamento de Engenharia Civil da Universidade Estadual de Maringá, pelos serviços de desenhista.

\section{REFERÊNCIAS BIBLIOGRÁFICAS}

Alencar, J.E. Profilaxia do calazar no Ceará, BrasiL. Rev. Inst. Med. Trop. S. Paulo, v. 3, n 4, p.175-180, 1961.

Alencar, J.E. Expansão do calazar no Brasil. Ceará méd., v.5, n.1/2, p.86-120, 1983.

Azevedo, A.C.R.; Rangel, E.F.; Costa, E.M.; David, J; Vasconcelos A.W.; \& Lopes, U.G. Natural infection of L. (Nyssomyia) whitmani (Antunes \& Coutinho,1939) by Leishmania of the braziliensis complex in Baturite, Ceará State, Northeastern BraziL. Mem. Inst. Oswaldo Cruz, v. 85, n. 2, p.251, 1990a.

Azevedo, A.C.R.; Rangel, E.F.\& Queiroz, Rg. $L$ migonei (França, 1920) naturally infected with peripylarian flagellates in Baturité, a focus of cutaneous leishmaniasis in Ceará State, Brazil. Mem. Inst. Oswaldo Cruz, v. 85, n. 4, p.479, 1990b.

Brandão-Filho, S.P.; Carvalho, F.G.; Brito, M.E.F.; Almeida, F.A.; Nascimento, L.A. American cutaneous leishmaniasis in Pernambuco, Brazil: eco-epidemiological aspects in "Zona da Mata"region. Mem. Inst. Oswaldo Cruz, v. 89, n. 3, p.445-449, 1994.

Deane, L.M.; Deane, M.P. \& Alencar, J.E. Observações sobre o combate ao
Phlebotomus longipalpis pela dedetização domiciliária em focos endêmicos de calazar, Ceará. Rev. bras. Malar., v. 7, p.131-141, 1955.

Falcão, L.R. Um novo modelo de armadilha luminosa de sucção para pequenos insetos. Mem. Inst. Oswaldo Cruz, v. 76, n. 3, p.:303305, 1981.

Hertig, M \& Fairchild, G.B. The control of Phlebotomus in Peru with DDT. Am. J. Trop. Med., v. 28, p. 207-230, 1948.

Lonardoni, M.V.C.; Teodoro, U; Arraes, S.M.A.A.; Silveira, T.G.V.; Bertolini, D.A.; Ishikawa, E.A.Y.; Shaw, J.J. Nota sobre leishmaniose canina no noroeste do Estado do Paraná, sul do BrasiL. Rev. Saúde Pública, v. 27, n. 5, p..378-379, 1993.

Magalhães, P.A.; Mayrink, W.; Costa, C.A.; Batista, S.M.; Michalik, M.S.M.; Williams, P. Calazar na zona do Rio Doce - Minas Gerais. Resultados de medidas profiláticas. Rev. Inst. Med. Trop. S. Paulo, v. 22, p.197202, 1980.

Ministério Da Saúde (Sucam). Relatório da Reunião Técnico Operacional Sobre o Controle da Leishmaniose Tegumentar Americana. Brasília, 1988. 17p.

Ministério Da Saúde (Fundação Nacional De Saúde). Casos notificados de leishmaniose tegumentar americana. Residentes na UF, por ano, segundo Unidade Federada. Brasil 1980 a 1994. SL.: s.n.,[1995?].

Ministério Da Saúde (Fundação Nacional De Saúde. Número de casos notificados de leishmaniose tegumentar americana. Residentes na UF, segundo Unidade Federada, Brasil - 1994. S.L.: s.n., [1996?]. Mimeogr.

Nery-Guimarães, F. \& Bustamante, F.M.. A aplicação domiciliária de DDT como base da profilaxia das leishmanioses. Estudo de um foco de leishmaniose muco-cutâneo cinco anos depois de aspersão periódica com aquele inseticida. Rev. bras. Malar., v. 6: p.127-130, 1954.

Queiroz, R.G.; Vasconcelos, I.A.B.; Vascocelos, A.W.; Pesssoa, F.A.C.; Souza, R.N. \& David, R. Cutaneous leishmaniasis in Ceara in Northeastern Brazil: incrimination of Lutzomyia whitmani (Diptera: Psychodidae) as a vector of Leishmania braziliensis in Baturité municipality. Am. J. Trop. Med. Hyg., v. 50, n. 6, p.693-698, 1994. 
Rosa, A.C.; Cuba, C.C.; Vexenat, A.; Barreto, A.C. \& Marsden, P.D. Predominance of Leishmania braziliensis braziliensis in the regions of Três Braços and Corte da Pedra, Bahia, BrasiL. Trans. roy. Soc. Trop. Med. Hyg., v. 82, p.409-410, 1988.

Silveira, T.G.V.; Teodoro, U.; Lonardoni, M.V.C.; Toledo, M.J.O.; Vedovello, D.; Bertolini, D.A.; Arraes, S.M.A.A. \& Guilherme, A.L.F. Investigação sorológica em cães de área endêmica de leishmaniose .tegumentar, no Estado do Paraná, Sul do Brasil. Cad Saúde Pública, v. 12, n. 1, p.8993, 1996.

Silveira, T.G.V.; Teodoro, U.; Lonardoni, M.V.C.; Guilherme, A.L.F.; Toledo, M.J.O.; Ramos, M.; Arraes, S.M.A.A.; Bertolini, D.A.; Spinosa, R.P. \& Barbosa, O.C. Aspectos epidemiológicos da leishmaniose tegumentar em área endêmica do Estado do Paraná, Brasil. Cad. Saúde Pública, v. 12, n. 2, p.141-147, 1996.

Sherlock, I.A. \& Almeida, S.P. Observações sôbre calazar em Jacobina, Bahia. V. Resultados de medidas profiláticas. Rev. bras. Malar., v. 22, n 1, p.175-182,1970.
Teodoro, U. Características ecológicas de flebotomíneos (Diptera, Psychodidae) em habitats antrópicos, município de Jussara, Paraná, Brasil. Curitiba, 1995. (Tese de Doutorado - Universidade Federal do Paraná)

Teodoro, U.; La Salvia Fo. V.; Lima, E.M.; Spinosa, R.P.; Barbosa, O.C.; Ferreira, M.E.M.C. \& Lonardoni, M.V.C. Observações sobre o comportamento de flebotomíneos em ecótopos florestais e extraflorestais, em área endêmica de leishmaniose tegumentar americana, no norte do Estado do Paraná, sul do BrasiL. Rev. Saúde Pública, v. 27, n. 4, p.242-249, 1993.

Vasconcelos, I.A.B.; Vasconcelos, A.W.; Fe Filho, N.M.; Qeurioz, R.G.; Santana, E.W.; Bozza, S.M.; Sallenave, S.M.; Valim, C. \& David, R. \& Lopes, U.G. The identity of Leishmania isolated from sand flies and vertebrate hosts in a major focus of cutaneous leishmaniasis in Baturité, Northeastern Brazil. Am. J. Trop. Med. Hyg., v. 50, n. 2, p.158-164, 1994.

Received: December 18, 1997; Revised: June 22, 1998; Accepted: October 26, 1998. 